\title{
An Effect of the Elastic Energy Stored in the Muscle-Tendon Complex at Two Different Coupling-Time Conditions during Vertical Jump
}

\author{
Sukwon Kim \\ Department of Physical Education, Research Institute of Physical Education, Chonbuk National University, \\ Jeonju, South Korea \\ Email: rockwall@jbnu.ac.kr
}

Received October $9^{\text {th }}$, 2012; revised November $12^{\text {th }}$, 2012; accepted November $24^{\text {th }}, 2012$

\begin{abstract}
A study was performed to evaluate effects of elastic energy stored pre-stretch on the mechanical work output while performing vertical jump. Eight male volunteers of age between 21 - 29 years old participated in the present study. The present study hypothesized that the vertical jump height during countermovement jumps (CMJ) would be higher than that during squat jump (SJ). During squat jump, a volunteer paused 0.5 sec before making upward movement. The results showed that ground reaction forces and vertical jump displacements were higher in CMJ in comparison to SJ. The study concluded that part of the positive work measured did derive from the recoil of the elastic component of the muscle-tendon complex.
\end{abstract}

Keywords: Coupling-Time; Vertical Jump; Elastic Energy

\section{Introduction}

During jumping, walking, or running, which causes the vertical displacement of the mass center of the body, the legs continue to flex and extend the joints in the lower extremities, such as hips, knees, and ankles (Young, 2003; Cavagna et al., 1971; Bobbert \& Schenau, 1988; Zajac et al., 2002). The mechanical analysis of patterns of walking, running, and jumping has been researched at a level of the lower extremities (Voigt et al., 1995; Bobbert et al., 1986; Umberger, 1998). However, the effects of the elastic energy stored and used in the muscle-tendon complex during vertical jumping have not been thoroughly explained (Young, 2003; Cavagna et al., 1971; Bobbert \& Schenau, 1988; Zajac et al., 2002; Voigt et al., 1995; Bobbert et al., 1986).

Without the combination of flexion and extension of the joints, humans could not walk, run, or jump properly (Voigt et al., 1995; Bobbert et al., 1986). During jumping, walking, and running, flexion of the legs results in stretching of the muscles, like the vastus lateralis, gluteus maximums, and gastrocnemius and tendons, like Achilles tendon. Many scientists (Komi, 2000; Morgan et al., 2000; Edman \& Tsuchiya, 1996; Ettema et al., 1990; Lee \& Herzog, 2002; Bosco et al., 1987; Morgan et al., 2000; De Ruiter et al., 2000) have thought that stretching of the muscle-tendon complex would cause the recoil of elastic energy, which produces a certain amount of mechanical work without utilization of chemical energy. This stored elastic energy is reused for the next following concentric contraction. However, the correlation between pre-stretch of the muscle-tendon complex in the lower extremity and vertical jump height has been a very sophisticated and disputed fact since the 19th century.

The goal of the present study was to evaluate effects of stored elastic energy in muscle-tendon complex in the lower extremity on mechanical work output represented by the height of the vertical jump. The experimental data is collected at two different coupling time conditions: 1) countermovement jump (CMJ); and 2) Squat jump (SJ).

The present study hypothesized that the vertical jump height during countermovement jumps would be higher than the vertical jump height during squat jump.

\section{Methods}

\section{Subject}

Eight participants were recruited from the general university population to participate in this study. All participants were ranged in age between 21 - 29 years old. They were normally involved in at least one physical activity that requires jumping or hopping. In order for subjects to be familiar with jumping elements, the participants were recruited depending on familiarity with jumping.

Each participant completed an informed consent form approved by the University's Internal Review Board (IRB). They had no history of lower extremity injury, surgery, or discomfort in the lower extremities. If they were injury free, they were qualified to continue the test. Otherwise, the participants were removed from the experiment. Social characteristics, such as ethnic background, were not considered.

\section{Instrumentation}

Kinematic and kinetic data were collected during each jump trial. Kinematic data were collected in 2 dimensions using a Motion Analysis Corporation video system. One NEC camera was set at a height of $80 \mathrm{~cm}$ and a distance of 7 meters from the force platform (Figure 1). The subject was filmed in the sagittal plane as they performed the countermovement and squat jumps. It recorded a field of view $304.5 \mathrm{~cm} \times 228.5 \mathrm{~cm}$ (Figure 2). The camera was leveled using a bubble level. In order to 


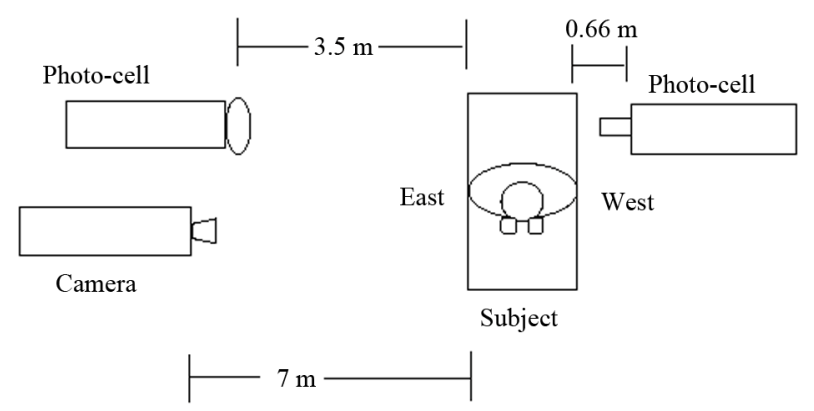

Figure 1.

Motion analysis equipment set-up.

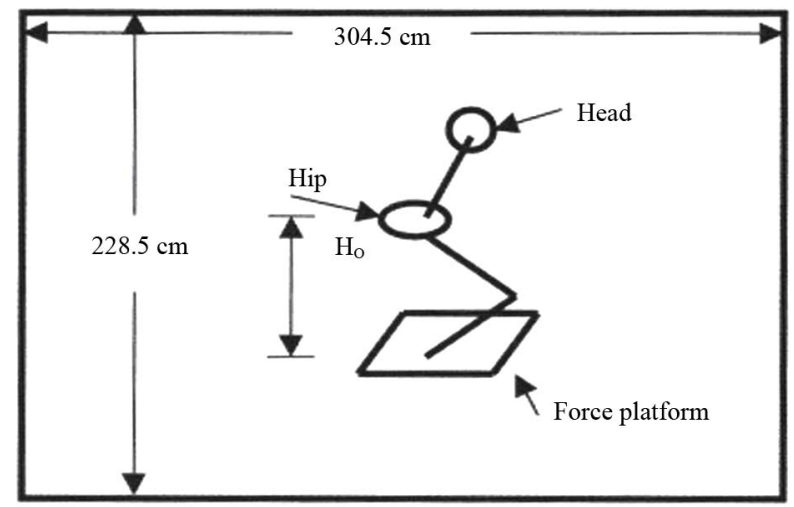

Figure 2.

Fixation of $\mathrm{H}_{0}$ for CMJ and SJ by the photo-cell (Figure 4) and a field of view.

optimize the image of the 5 reflective markers and the subjects, the flood light was set behind the camera; one flood light was used to enhance the brightness of the testing area to optimize digitization process. The reflective markers were placed over the skin at the position of five bony prominences (Figure 3); the head of the fifth metatarsophalangeal, the lateral malleolus, the lateral epicondyle, the greater trochanter, and the glenohumeral joint. The four segments (Figure 3) were classified; Upper body (between the glenohumeral joint and the greater trochanter), Thigh (between the greater trochanter and the lateral epicondyle), Shank (between lateral epicondyle and the lateral malleolus) and Foot (between the head of the fifth metatarsophslangeal and the lateral malleolus).

The reflector was set to stand 0.66 meter away from the west edge of the force platform and the projector was set to stand 4.15 meters away from the east edge of the force platform (Figure 1). It made a beep sound when the light to the reflector was disturbed by an object; in this case, hip was the only one that disturbed the light (Figure 4); the subjects controlled the squatting height for the CMJ and SJ by themselves by jumping after a beep sound. The use of the photo-cell was designed by the investigator to control and equalize the squat height during the countermovement jump and the squat jump (Figures 2 and 4).

Kinetic data was collected on an AMTI force platform (AMTI Inc. Model OR6-5-1). Force data in the vertical (Fz) direction were collected at $1200 \mathrm{~Hz}$ using the Ariel Performance Analysis system (APAS). An AMTI signal amplifier (Model SGA6-3) was used to amplify the analog signal before it reached the APAS. Kinetic data were collected for 3 seconds,

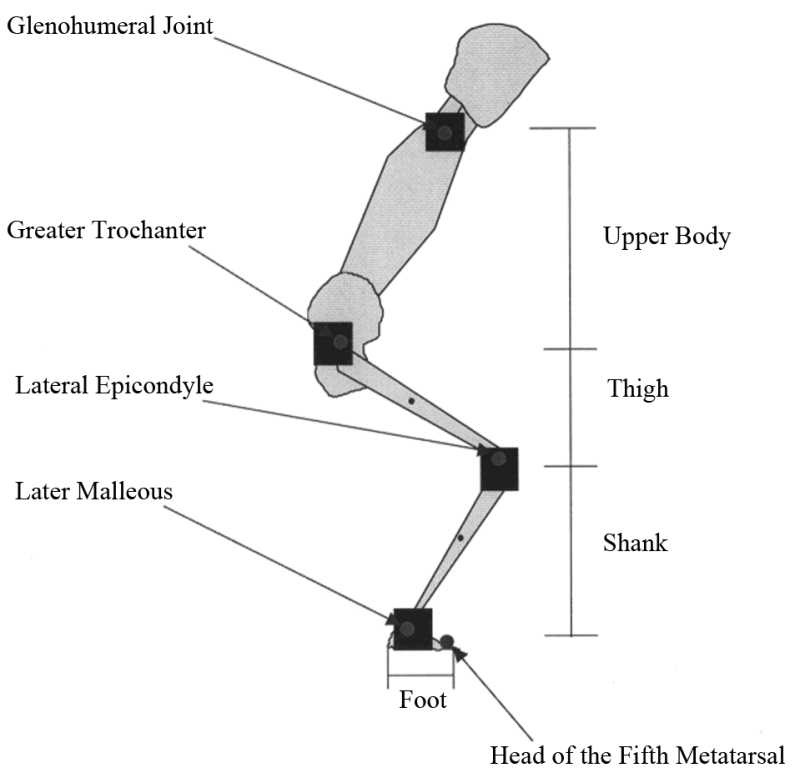

Figure 3.

Placement of the reflective markers and classification of four segments.

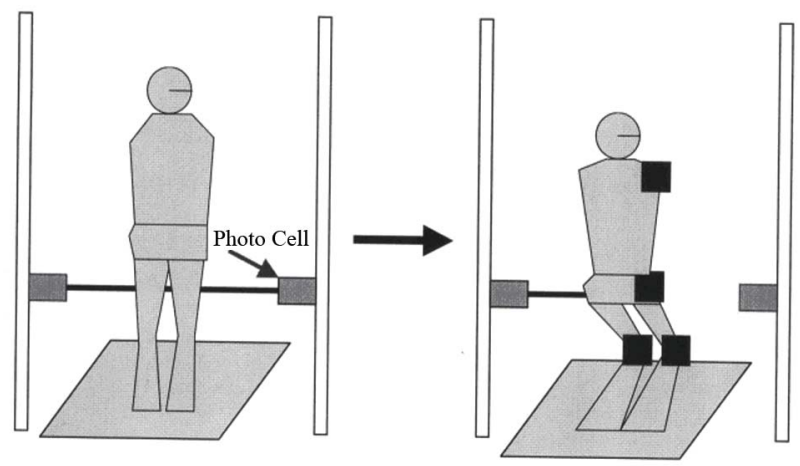

Figure 4.

Control of the squat height by the photo-cell.

recording 90 frames for each jump. The ground contact period at the lift-off during a pause and the peak ground reaction forces during landing were monitored for general consistency throughout the whole experiment. A trial was considered unacceptable if the subject did not contact the ground with both feet simultaneously, making two impact peaks, or if the ground contact period at the lift-off is less than 0.5 second as lift-off during squat jump.

Kinematic data were recorded for the same period by the Motion analysis Corporation video system and transferred to HU-M-AN software for digitizing. The digitization process was processed manually by clicking the mouse over the reflective markers on the computer screen. The distortion rate and the distance rate were measured using $1 \mathrm{~m} \times 1 \mathrm{~m}$ square and 1.905 meters stick bar. HU-M-AN was used to calculate kinematic variables.

\section{Experimental Protocol}

The participants were asked to wear the shoes that he/she wore during his/her physical activities. For the clear view of the reflective markers, the participants were asked to wear short 
pants and shirts without arm sleeves. Before the actual experiments started, several practices were given to each subject on the force platform in order for them to be familiar with the test process. For example, the squat height had to be very same during CMJs and SJs for each participant to equalize the potential energy during each jump. For this equalization, the investigator used one set of the photo-cell (Figure 4) to match the squat height in CMJs and SJs. Therefore, all participants had to jump after hearing a beep sound from the photo-cell (Figures 2 and 4). The photo-cell was also used to fix the relative knee angle. The investigator inspected the ground reaction force data collected by the APAS system during the practice trials to determine jump consistency. Feedback was given to the subjects to help them improve their jump quality and consistency. All subjects were given as many practice jumps as they wanted.

For the actual experiment, subjects were asked to stand on the force platform in the sagittal plane from the camera. For all jumps, the subjects were instructed to have arms cross over the chest to minimize the effect of arm swing. For all jumps, each subject began from a relaxed standing position and was instructed to jump as high as possible. For the countermovement jumps (CMJs), subjects were instructed to extend (jump) vertically hip, knee, and ankle joints after flexing them without a delay between flexion and extension of each joint. For the squatting jumps (SJs), each subject began from the standing position, and was instructed to squat and wait for 0.5 second before jumping. Each subject performed the five counter-movement jump (CMJs) and five squat jumps (SJs) alternately with about 50 - 60 second break between each jump.

Ground reaction force data collected for each subject were divided by their body weight in order to express all kinetic data in terms of body weight. When the 10 jumps had been completed, the investigator asked the participants about the fatigue level in the lower extremities to assess the consistency of all the jumps. If a subject' ability to do 10 jumps in a row with the break was not consistent, the investigator had given longer break between each jump.

\section{Data Analysis}

Descriptive and inferential statistical analyses were performed by utilizing the SPSS (IBM Corporation, USA). The differences were evaluated using paired t-tests. The results were considered as statistically significant when $p \leq 0.05$.

\section{Results}

The goal of the present study was to compare the influence of stored elastic energy in muscle-tendon complex in the lower extremity on mechanical work out put represented by the height of the vertical jump between Counter Movement Jump (CMJ) and Squat Jump (SJ). The comparison in the peak ground reaction force and the vertical displacement of vertical jump between CMJ and SJ was made. Relative knee angle was measured to see if there was a movement of segments during the pause phase.

\section{Relative Knee Angle}

The relative knee angle was measure in order to observe if the pause between flexion and extension during SJ was correctly controlled. Figure $\mathbf{5}$ illustrates the relative knee angle typical for SJ. There was no significant change in relative knee

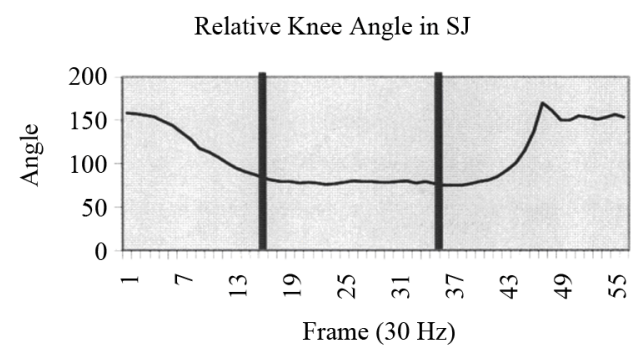

Figure 5.

Example of relative knee angle curve during squat jump.

angle during the pause phase in $\mathrm{SJ}$ for more than 0.5 seconds (15 frames), which indicates that the pause was controlled successfully during SJ. The mean relative knee angle in Figure 5 between the two vertical lines (a pause) was 78.41 \pm 1.18 .

\section{Peak Ground Reaction Force}

The peak ground reaction force was measured in each subject in order to compare the ground reaction force exerted during push-off between during CMJ and during SJ. Table 1 reports the mean peak GRF during CMJ and SJ. In all subjects except subject 1 , mean of Peak GRF in CMJ was significantly higher than mean of Peak GRF in SJ. For example, in Subject 6, the Mean Peak GRF in the SJ was $1835 \pm 56.85$ and in the CMJ, it was $2031.25 \pm 57.09$. In addition, paired t-test of individual mean peak ground reaction force of CMJ and SJ $(t=4.449, d f=$ $6, P=0.0022)$ suggested that mean peak ground reaction forces of CMJ was significantly higher than that of SJ.

\section{Initial Height (Ho)}

The most important different in the methodology of this study from the other studies was that the investigator tried to control the stored elastic potential energy by equalizing the initial height at the push-off during both countermovement and squat jumps. The set-up for Ho was the most important process in the study since the photo-cell was to control Ho which represented the stored elastic potential energy. Table 2 illustrates Ho in both CMJ an SJ. Paired t-test showed that the values of Ho in CMJ and SJ were statistically insignificant $(t=0.535, d f$ $=7, P=0.61$ ). This result suggested that the values of Ho were very close to each other suggesting that the stored elastic potential energy at Ho was said to be the same.

\section{Vertical Jump Displacement}

The vertical jump height in each jump was measure to observe the influence of stored elastic energy in muscle-tendon complex in the lower extremity on mechanical work output represented by the height of the vertical jump. All five jumps in each condition which was Squat jump and Countermovement jump was averaged out.

Figure 6 illustrates the comparison of vertical displacement between Squat Jump and Countermovement Jump. Paired t-test showed significantly higher displacement in CMJ in compareson to SJ $(t=4.26, d f=7, P=0.004)$. The most increase was performed in Subject 3. However, the Subject 1 jumped just $0.3 \%$ higher during CMJ. Table 3 demonstrates the percentage increase in the vertical displacement of CMJ in comparison to the vertical displacement of SJ. 
Table 1.

Mean peak ground reaction force (N) in CMJ and SJ.

\begin{tabular}{cccc}
\hline & CMJ & SJ & $\begin{array}{c}\text { T-test } \\
(\boldsymbol{P} \text {-value) }\end{array}$ \\
\hline Subject 1 & $1835.25 \pm 12.47$ & $1866 \pm 24.40$ & 0.32 \\
Subject 2 & $1350 \pm 31.50$ & $1480 \pm 35.50$ & 0.0003 \\
Subject 3 & $1265 \pm 6.20$ & $1460 \pm 49.22$ & 0.0001 \\
Subject 4 & $1727.5 \pm 55.22$ & $1827 \pm 54.5$ & 0.02 \\
Subject 5 & $1835 \pm 29.55$ & $1905 \pm 53.07$ & 0.05 \\
Subject 6 & $1834.25 \pm 56.85$ & $2031.25 \pm 57.09$ & 0.0006 \\
Subject 7 & $1303.5 \pm 25.3$ & $1497.75 \pm 21.09$ & 0.0001 \\
Subject 8 & $1420 \pm 35.30$ & $1460.75 \pm 5.20$ & 0.037 \\
\hline
\end{tabular}

Table 2.

Average Ho (initial height (cm)) of CMJ and SJ.

\begin{tabular}{ccccccccccc}
\hline Subject & $\mathbf{1}$ & $\mathbf{2}$ & $\mathbf{3}$ & $\mathbf{4}$ & $\mathbf{5}$ & $\mathbf{6}$ & $\mathbf{7}$ & $\mathbf{8}$ & Mean & SD \\
\hline CMJ & 46.84 & 57.30 & 55.58 & 50.38 & 47.20 & 56.92 & 53.50 & 56.20 & 52.99 & 4.30 \\
SJ & 49.00 & 55.95 & 53.60 & 54.78 & 55.95 & 53.30 & 53.27 & 54.29 & 53.76 & 2.20
\end{tabular}

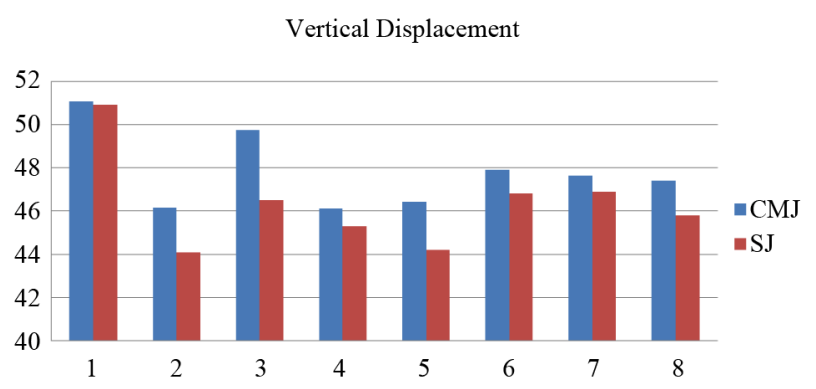

Figure 6.

Comparison of vertical displacement between squat jump and countermovement jump.

Table 3.

Percentage increase in CMJ in comparison to SJ in vertical displacement.

\begin{tabular}{ccccccccc}
\hline Subject & $\mathbf{1}$ & $\mathbf{2}$ & $\mathbf{3}$ & $\mathbf{4}$ & $\mathbf{5}$ & $\mathbf{6}$ & $\mathbf{7}$ & $\mathbf{8}$ \\
\hline & $0.3 \%$ & $4.7 \%$ & $7.0 \%$ & $1.8 \%$ & $5.0 \%$ & $2.4 \%$ & $1.6 \%$ & $3.5 \%$ \\
\hline
\end{tabular}

\section{Discussion}

The goal of the present study was to evaluate the influence of stored elastic energy in muscle-tendon complex in the lower extremity on mechanical work output represented by the height of the vertical jump.

\section{Stretching of and Potential Energy Created in Actin and Myosin Filaments}

In life, muscle fibers can be stretched up to $10 \%$ of their length (Biewener et al., 1981). These large stretches are used to gain optimal mechanical energy in muscles and elastic fibers. The enhancement of performance during concentric contraction followed by eccentric contraction can be explained by these stretching (Voigt et al., 1995; Komi, 2000; Lee \& Herzog, 2002; Morgan et al., 2000; Bullimore et al., 2008; Hodson-Tole \&
Wakeling, 2010). In the present study, the initial height and relative knee angle of each individual right before the take-off for vertical jump was set to a fixed height for both trials (CMJ and SJ) by utilizing the photo cell. If not controlled, greater force could be produced in one case resulting in higher jump displacement (Voigt et al., 1995). Active muscle resists stretching with a force greater than the isometric force (Lee \& Herzog, 2002; Bosco et al., 1987; Bullimore et al., 2008; Cavagna et al., 1985; Herzog \& Leonard, 1997; Meijer et al., 1997). The force attained after large, fast stretches was due to 1) a greater force developed by each bridge (Hodson-Tole \& Wakeling, 2010); or 2) an increased number of cross-bridge attachments (Lee \& Herzog, 2002; Proske \& Morgan, 1999). The decrease in the number of attached cross-bridges reduced the load-sustaining ability of the fibers to result in the initial rapid lengthening. However, the detached cross-bridges start to re-attach to the thin filaments. The increase in the number of attached crossbridges restored the load-sustaining ability to cause the subsequent shortening when $\mathrm{F}$ (instaneous force) is not much larger than Fo (maximal force at zero velocity).

\section{Power and Performance Enhancement by Stored Elastic Energy in the Muscle-Tendon Complex and the Coupling Time}

In agreement with the previous studies (Ettema et al., 1990; Lee \& Herzog, 2002; Bullimore et al., 2008; Cavagna et al., 1985) peak ground reaction forces (i.e. concentric contraction) before take-off and vertical displacement in the present study were higher in CMJ than in SJ. This result may confirm that, during eccentric contraction, elastic energy due to stretching could be stored in the muscle-tendon complex for later use such as concentric contraction, but, a coupling time could be a limiting factor because stored elastic energy would dissipate rapidly. The force-velocity relationship suggests that the force generated by a muscle is a function of its velocity (Lieber, 2002). For example, force drops precipitously as muscle is allowed to shorten. Nevertheless, the eccentric phase during flexion of the lower limbs is considered to enhance performance in the concentric phase. Cavagna et al. (1971) found that elastic energy stored in muscle-tendon complex would produce power enhancement during sprint running even after the force was supposed to drop. Studies (Komi, 2000; Komi \& Nicol, 2000) found that the concentric phase without the eccentric phase generated less ground reaction force than the concentric phase following by the eccentric phase. However, studies found that the period (i.e. coupling time) between the two contractions was a key concern. Bosco and Rusko (2008) presented a study of the coupling time during treadmill running at different speeds using special soft shoes in addition to normal shoes. The study measured the oxygen consumption to demonstrate the energy consumed with soft shoes and normal shoes. The assumption was that during running, because cushioning decreased the force between colliding bodies by increasing the time collision, soft shoes would increase the coupling time, costing more energy. As assumed, the results showed that running with soft shoes required greater energy consumption than running with normal shoes. Their results suggested the effect of coupling time as a limiting factor for recoil of elastic energy which means that longer the coupling time, less possibility to re-use the elastic energy stored in the muscle-tendon complex.

Also, the present study suggested that skeletal muscles could 
be more efficient when using stored elastic energy in muscletendon complex. In agreement with previous findings (Voigt et al., 1995; Bosco \& Rusco, 2008), the CMJ showed better performance results than SJ. During SJ conditions, concentric contraction took place $0.5 \mathrm{sec}$ after the stretching. This suggested that $0.5 \mathrm{sec}$ was enough to dissipate the elastic energy in muscle-tendon complex during vertical jump. Greater positive mechanical work due to greater ground reaction force during pushoff and vertical displacement suggested that the energy stored was used to produce mechanical work during concentric contraction.

The conclusion was that part of the positive work measured did not derive from transformation of chemical energy but it was derived, without cost of chemical energy, from the recoil of the elastic component of the muscle-tendon complex.

Assumptions

1) Muscle can absorb, store, and reutilize the potential energy.

2) During the negative work phase of the vertical jump, extensor muscles are stretched and elastic energy, produced while stretching of the muscles, is stored in the muscle-tendon complex and reutilized during the positive work phase.

3) Potential energy stored after stretching is used to enhance the performance during shortening from a state of isometric contraction.

4) 0.5 seconds delay during $\mathrm{SJ}$ is a long enough time for the stored elastic energy to dissipate in heat.

5) Power produced in the lower extremity during the positive work phase is proportionally related to vertical velocity.

6) An increased power (force $\times$ velocity) increases jump height.

\section{Acknowledgements}

This paper was supported by research funds of Chonbuk National University in 2012.

\section{REFERENCES}

Biewener, A., Alexander, R., \& Heglund, R. (1981). Elastic energy storage in the hopping of kangaroo rats. Journal of Zoology, 195, 369383. doi:10.1111/j.1469-7998.1981.tb03471.x

Bobbert, M., Huijing, P., \& Ingen Schenau, G. (1986). An estimation of power output and work done by the human triceps surae muscletendon complex in jumping. Journal of Biomechanics, 19, 899-906. doi:10.1016/0021-9290(86)90185-5

Bobbert, M., \& van Ingen Schenau, G. J. (1988). Coordination in Vertical Jumping. Journal of Biomechanics, 21, 249-262. doi:10.1016/0021-9290(88)90175-3

Bosco, C., \& Rusko, H. (2008). The effect of prolonged skeletal muscle stretch-shortening cycle on recoil of elastic energy and on energy expenditure. Acta Physiologica, 119, 219-224.

Bosco, C., et al. (1987). The effect of pre-stretch on mechanical efficiency of human skeletal muscle. Acta Physiol Scand, 131, 323-329. doi:10.1111/j.1748-1716.1987.tb08246.x

Bullimore, S., MacItosh, B., \& Herzog, W. (2008). Is a parallel elastic element responsible for the enhancement of stead-state muscle force following active stretch? The Journal of Experimental Biology, 211,
3001-3008. doi:10.1242/jeb.021204

Cavagna, G., Komarek, L., \& Mazzoleni, S. (1971). The mechanism of sprint running. Journal of Physiology, 281, 139-155.

Cavagna, G., Mazzanti, M., Heglund, N., \& Citterio, G. (1985). Storage and release of mechanical energy by active muscle: A non-elastic mechanism? Journal of Experimental Biology, 115, 79-87.

De Ruiter, C., Didden, W., Jones, D., \& De Haan, A. (2000). The force-velocity relationship of human adductor pollicis muscle during stretch and the effects of fatigue. Journal of Physiology, 526, 671681. doi:10.1111/j.1469-7793.2000.00671.x

Edman, K., \& Tsuchiya, T. (1996). Strain of passive elements during force enhancement by stretch in frog muscle fibres. Journal of Physiology, 490, 191-205.

Ettema, G., Van Soest, A., \& Juijing, P. (1990). The role of series elastic structures in prestretch-induced work enhancement during isotonic and isokinetic contractions. Journal of Experimental Biology, 154, 121-136.

Herzog, W., \& Leonard, T. (1997). Depression of cat soleus forces following isokinetic shortening. Journal of Biomechanics, 30, 865-872. doi:10.1016/S0021-9290(97)00046-8

Hodson-Tole, E., \& Wakeling, J. (2010). The influence of strain and activation on the locomotor function of rat ankle extensor muscles. The Journal of Experimental Biology, 213, 318-330. doi:10.1242/jeb.031872

Komi, P., \& Nicol, C. (2000). Stretch-shortening cycle fatigue. In B McIntosh, \& B. Nigg (Eds.), Biomechanics and biology of movement. Champaign, IL: Human Kinetics Publishers.

Komi, P. (2000). Stretch-shortening cycle: A powerful model to study normal and fatigued muscle. Journal of Biomechanics, 33, 11971206.

Lee, H., \& Herzog, W. (2002). Force enhancement following muscle stretch of electrically stimulated and voluntarily activated human adductor pollicis. The Journal of Physiology, 545, 321-330. doi:10.1113/jphysiol.2002.018010

Lieber, L. R. (2002). Skeletal muscle structure and function, \& plasticity. Baltimore, MD: Lippincott Williams \& Wilkins.

Meijer, J., Grootenboer, B., \& Koopman, P. (1997). Fully isometric length-force curves of rat muscle differ from those during and after concentric contractions. Journal of Applied Biomechanics, 13, 164181.

Morgan, D., Whitehead, N., Wise, A., Gregory, J., \& Proske, U. (2000). Tension changes in the cat soleus muscle following slow stretch or shortening of the contracting muscle. Journal of Physiology, 522, 503-513. doi:10.1111/j.1469-7793.2000.t01-2-00503.x

Proske, U., \& Morgan, D. (1999). Do cross-bridge contribute to the tension during stretch of passive muscle? Journal of Muscle Research and Cell Mobility, 20, 433-442. doi:10.1023/A:1005573625675

Umberger, B. (1998). Mechanics of the vertical jump and two-joint muscles: Implications for training. Strength and Conditioning, 20, 70-74. doi:10.1519/1073-6840(1998)020<0070:MOTVJA>2.3.CO;2

Voigt, M., Simnsen, E., Dyhre-poulsen, P., \& Klausen, K. (1995). Mechanical and muscular factors influencing the performance in maximal vertical jumping after different pre-stretch load. Journal of Biomechanics, 28, 293-307. doi:10.1016/0021-9290(94)00062-9

Young, W., \& Behmm, D. (2003). Effects of running, static stretching and practice jumps on explosive force production and jumping performance. The Journal of Sports Medicine and Physical Fitness, 43, 21-27.

Zajac, F., Neptune, R., \& Kautz, S. (2002). Biomechanics and muscle coordination of human walking: Part I: Introduction to concepts, power transfer, dynamics and simulations. Gait and Posture, 16, 215232. doi:10.1016/S0966-6362(02)00068-1 